\title{
Higher-order breathers as quasi-rogue waves on a periodic background
}

\author{
Omar A. Ashour - Siu A. Chin . \\ Stanko N. Nikolić • Milivoj R. Belić
}

Received: 4 March 2021 / Accepted: 22 December 2021 / Published online: 17 January 2022

(C) The Author(s) 2022

\begin{abstract}
We investigate higher-order breathers of the cubic nonlinear Schrödinger equation on a periodic elliptic background. We find that, beyond first order, any arbitrarily constructed breather on a disordered background generates a single-peaked solitary wave. However, on the periodic backgrounds, the so-called quasi-rogue waves are found more common. These are the quasiperiodic breathers that feature distorted side peaks. We construct such higher-order breathers out of constituent first-order breathers with commensurate periods (i.e., as higher-order harmonic waves). In addition to quasiperiodic, we also find fully periodic breathers, when their wavenumbers are harmonic multiples of the background and each other. But they are truly rare, requiring finely tuned parameters. Thus,
\end{abstract}

Supplementary Information The online version contains supplementary material available at https://doi.org/10.1007/ s11071-021-07172-x.

\section{O. A. Ashour ( $\square)$}

Department of Physics, University of California, Berkeley,

CA 94720, USA

e-mail: ashour@berkeley.edu

\section{S. A. Chin}

Department of Physics and Astronomy, Texas A\&M

University, College Station, TX 77843, USA

S. N. Nikolić

Institute of Physics Belgrade, University of Belgrade,

Pregrevica 118, Belgrade 11080, Serbia

S. N. Nikolić · M. R. Belić

Science Program, Texas A\&M University at Qatar, P.O. Box 23874, Doha, Qatar on a periodic background, we arrive at the paradoxical conclusion that the higher-order quasi-rogue waves are rather common, while the truly periodic breathers are exceedingly rare.

Keywords Rogue waves - Breathers - Nonlinear Schrodinger equation

\section{Introduction}

The dimensionless cubic nonlinear Schrödinger (NLS) equation [1], given by

$i \frac{\partial \psi}{\partial x}+\frac{1}{2} \frac{\partial^{2} \psi}{\partial t^{2}}+\psi|\psi|^{2}=0$,

with $t$ and $x$ being the transverse and longitudinal variables and $\psi \equiv \psi(x, t)$ the slowly varying wave envelope, has been widely used in the field of nonlinear optics and photonics to guide experimental realizations [2-4] and theoretical explorations [5-11] of higher-order breathers and rogue waves (RWs) in optical fibers. For a recent review, see Ref. [12]. The physics of RWs is getting more attention since a new scheme to excite RWs, such as via electromagnetically induced transparency (EIT) [13-15], is proposed [16].

While the study of breathers and RWs on a uniform background, including those based on extended NLS equations, has matured by now, with a vast body of analytical results $[6,8,12,17-22]$, similar studies on periodic backgrounds have started only recently [9,23-25]. For clarity, at this place we provide a short outlook on 
the interconnection between different waveforms that are encountered in the field thus far. Foremost, to see interesting new waveforms, one needs a background. Without background, at most one can observe bright solitons $[1,12]$. Normally, breathers and RWs (higherorder breathers) are generated when one seeds the background with proper initial wave conditions, based on the Darboux transformation (DT). Such initial waves also provide conditions for the breather-to-soliton conversion, both bright and dark, which usually are difficult to observe in NLS equation without proper system preparation. Thus, starting a numerical experiment with uniform or randomly perturbed background and an arbitrary collection of Stokes waves will only occasionally, here and there, produce a second-order RW, almost never higher-order RWs. For observing a full wealth of different waveforms (breathers, solitons, RWs of different order, quasiperiodic and fully periodic RWs), in general one needs specially prepared nonuniform backgrounds and specially prepared initial waves. Previously, we considered backgrounds perturbed by the Jacobi elliptic functions (JEFs) and initial waves coming from Akhmediev breathers engineered using DT $[24,25]$. The fine point in the numerical procedure is how to prepare boundary conditions on the numerical window.

In this work, we are specifically interested in breathers of the simple cubic NLS equation on the dnoidal Jacobi elliptic function (JEF) background, which has the form:

$\psi_{0}(x, t)=\operatorname{dn}(t ; k) e^{i x\left(1-\frac{k^{2}}{2}\right)}$,

where $k$ is the elliptic modulus (not to be confused with the elliptic parameter, $k^{2}$ ). As our analysis will show below, at this time it is unlikely that one can derive analytical expressions for our breathers; therefore, we focus on studying solutions of (1) using a numerical implementation of the analytical DT procedure [7-11]. In a nutshell, the procedure states that given a simple, zeroth-order "seed" solution $\psi_{0}(x, t)$ of (1), such as (2), one can generate an $N$ th-order solution recursively via:

$\psi_{N}(x, t)=\psi_{0}(x, t)+\sum_{m=1}^{N} \frac{2 i r_{m 1} s_{m 1}^{*}\left(\lambda_{m}-\lambda_{m}^{*}\right)}{\left|r_{m 1}\right|^{2}+\left|s_{m 1}\right|^{2}}$,

where the sum goes over $N$ constituent first-order solutions of the Lax pair equation, characterized by generally complex eigenvalues $\lambda_{m}$. The two functions $r_{m 1}(x, t)$ and $s_{m 1}(x, t)$ are the Lax pair generating functions for the $m$ th first-order constituent breather. In this work, we restrict ourselves to purely imaginary eigenvalues:

$\lambda_{m}=i v_{m}, \quad$ with $0<v_{m}<1$,

corresponding to Akhmediev-type breathers [26], rather than Kuznetsov-Ma-type breathers [27,28]. As per the peak height formula (PHF) [23], these solutions have a peak height of

$\psi_{N}(0,0)=1+2 \sum_{m=1}^{N} v_{m}$.

In this work, we will concentrate on demonstrating the surprising finding mentioned in the abstract: Beyond first order, any arbitrarily constructed breather on an elliptic background looks very much like a rogue wave. But, to generate quasiperiodic and fully periodic RWs one needs to pay close attention how the periodicities of constituent waves are arranged over the available numerical window. We relegate all technical details on the DT and PHF to the appendix.

The paper is structured as follows. In Sect. 2, we demonstrate, by enforcing the reality of the wavenumber, that in contrast to the uniform background case $[17,18]$, the first-order breathers on an elliptic background can at most be quasiperiodic in the $t$-direction unless the matching condition (23) is obeyed. The concatenation of $N$ such first-order breathers then generally results in an aperiodic $N$ th-order breather greatly resembling a rogue wave (RW). In Sect. 3, we show that if the periods of the constituent first-order breathers are commensurate with one another, then one sees a quasiperiodic $N$ th-order breather, where the repeating side peaks are distorted by the background. In Sect. 4 , we show that truly periodic $N$ th-order breathers are produced if and only if the periods of the constituent first-order breathers are all matched to that of the background. This requires restricting the parameters $v$ and $k$ to a special set of contours $\gamma_{q}$ (Eq. 23) in the $v k$-plane. Our conclusions are summarized in Sect. 5.

\section{Aperiodic breathers on an elliptic background}

For a breather to be periodic (along the $t$ direction), its wavenumber must be real [9]. Assuming purely imag- 
inary eigenvalues $\lambda_{m}=i v_{m}$, the half-wavenumber is given by

$\kappa_{m}=\sqrt{\left(\lambda_{m}-\frac{k^{2}}{4 \lambda_{m}}\right)^{2}+1}=\sqrt{1-\left(v_{m}+\frac{k^{2}}{4 v_{m}}\right)^{2}}$.

For $\kappa_{m}$ to be real, we must have

$\left(v_{m}+\frac{k^{2}}{4 v_{m}}\right)^{2} \leq 1 \quad \Longrightarrow \quad k^{2} \leq 4 v_{m}\left(1-v_{m}\right)$,

which restricts the range of $v_{m}$ to

$\frac{1}{2}-\frac{1}{2} \sqrt{1-k^{2}} \leq v_{m} \leq \frac{1}{2}+\frac{1}{2} \sqrt{1-k^{2}}$.

Alternatively, from (7) one sees that

$k^{2} \leq 4\left[\frac{1}{2}+\left(v_{m}-\frac{1}{2}\right)\right]\left[\frac{1}{2}-\left(v_{m}-\frac{1}{2}\right)\right]$,

or

$k^{2}+\frac{\left(v_{m}-\frac{1}{2}\right)^{2}}{(1 / 2)^{2}} \leq 1$,

which then restricts $\left(v_{m}, k\right)$ to half of an ellipse $\Gamma$, centered on $(1 / 2,0)$, with the vertical semimajor axis being 1 and the horizontal semiminor axis $1 / 2$. This is shown in Fig. 6 and summarized succinctly by

$$
\begin{aligned}
& \left(v_{m}, k\right) \in \Gamma \\
& \quad:=\left\{\left(v_{m}, k\right) \in(0,1) \times[0,1] \mid k^{2}+\frac{\left(v_{m}-\frac{1}{2}\right)^{2}}{(1 / 2)^{2}} \leq 1\right\} .
\end{aligned}
$$

In Fig. 1, we show the three periods of a first-order breather obeying the $\kappa$-reality condition (11). This breather is not periodic since the repeating side peaks are not identical to the central peak. Next, in Fig. 2, we show a set of higher-order breathers. Their eigenvalues $v_{m}$ obey (11), but are otherwise arbitrary. These higherorder breathers are even more aperiodic, with the repeating side peaks highly distorted and diminished in intensity, leaving only a central peak of great height intact. It is a matter of definition how one defines a rogue wave, but Fig. 2 clearly shows four solitary, highintensity peaks amid an increasingly disordered background, each resembling more an oceanic rogue wave than a breather. We will refer to these unmatched solutions as quasi-rogue waves (QRWs). The phenomenon of QRW has only come to light recently in the study of higher-order breathers on periodic backgrounds $[25,29]$. They are not apparent in the study of first-order breathers on a periodic background, nor are they mentioned in the study of RWs on a constant background.

\section{Quasiperiodic breathers}

We now match the periods of the constituent breathers of these higher-order structures to the fundamental breather, i.e.:

$\kappa_{m}=m \kappa, \quad m=2,3, \ldots N$,

where $\kappa \equiv \kappa_{1}$ is the period of the fundamental breather, characterized by $v \equiv v_{1}$. We choose the constituent breather wave numbers to be harmonics of each other, analogously to the uniform background case where these breathers would have been strictly periodic and of maximal peak height (at a given periodicity) [18]. Writing out (12) explicitly gives

$$
\begin{aligned}
& m \sqrt{-\frac{k^{4}}{16 v^{2}}-\frac{k^{2}}{2}-v^{2}+1} \\
= & \sqrt{-\frac{k^{4}}{16 v_{m}^{2}}-\frac{k^{2}}{2}-v_{m}^{2}+1},
\end{aligned}
$$

which leads to an expression for $v_{m}$ in terms of $v$ and $k$

$$
v_{m}(k, v)=\frac{\sqrt{G_{m}(k, v)+\sqrt{\left[G_{m}(k, v)\right]^{2}-64 k^{4} v^{4}}}}{4 \sqrt{2} v},
$$

where

$$
\begin{array}{r}
G_{m}(k, v) \equiv k^{4} m^{2}+8\left(k^{2}-2\right)\left(m^{2}-1\right) v^{2} \\
+16 m^{2} v^{4} .
\end{array}
$$

A plot of Eq. (14) for multiple values of $m$ is shown in Fig. 3. Note that in Fig. 3, the curves $v_{m}(k)$, $\forall m \in \mathbb{Z}^{+}$, clearly intersect at a point $\left(v_{\max }, v_{\max }\right)$ in the $v v_{m}$-plane, given by the upper limit of Eq. (8):

$v_{\max }(k)=\frac{k^{\prime}}{2}+\frac{1}{2}$, 
Fig. 1 First-order breather with

$(v=0.8602, k=3 / 5) \in \Gamma$ (i.e., $\kappa \in \mathbb{R}$ ). The distortion of the side peaks is evident in the contour plot. In all contour plots that follow, maroon represents the background $(|\psi|=1)$, teal green is 0.25 above the background, while light orange is 0.2 below the background. The remaining colors denote other relevant low- $|\psi|$ features. The colormap is designed to showcase only the relevant low- $|\psi|$ features of the breathers, with a maximum height of 0.8 above the background
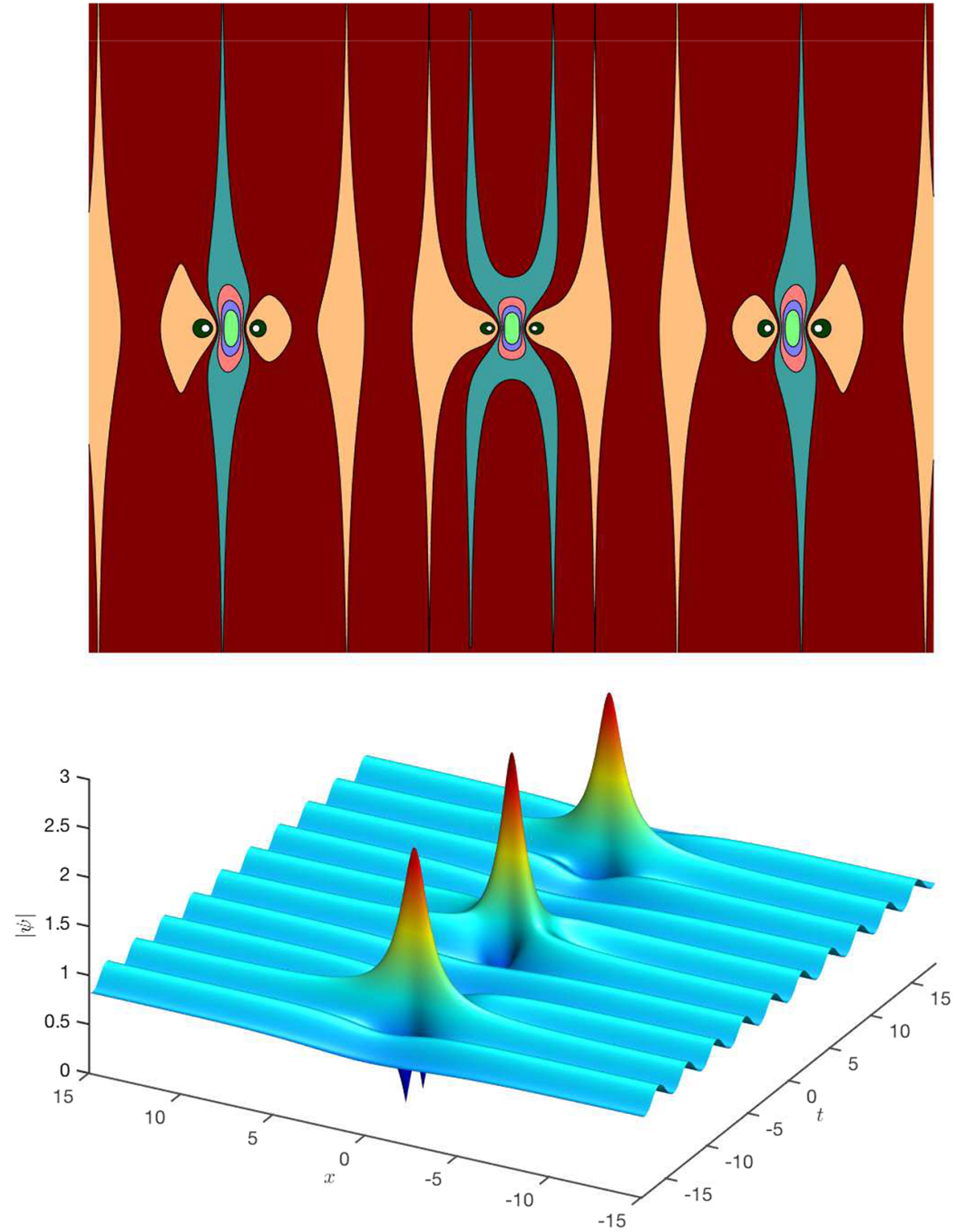

where $k^{\prime} \equiv \sqrt{1-k^{2}}$ is the complementary elliptic modulus. A first-order solution characterized by this value of $v$ is known as the concentrated cnoidal rogue wave (CCRW) [9] and was simply referred to as the "bright" rogue wave in Ref. [23]. This solution with $\kappa=0$ is a single solitary peak on a periodic background and is considered the natural generalization of the Peregrine rogue wave on a constant background. However, higher-order rogue waves generated by multiple values of (16) have degeneracy problems and cannot be described by the DT [10]. By contrast, as shown in Fig. 2, an arbitrary high-order QRW can be easily constructed using DT.

Figure 3, showing the plot of Eq. (14), implies that for a fixed $m$, there is some cutoff $v=v_{m}^{*}(k)$ such that $v_{l}\left(v=v_{m}^{*}, k\right) \notin \mathbb{R} \forall l>m$. Thus, to generate a breather of order at most $N$, one must have $v \in\left(v_{N}^{*}, v_{N+1}^{*}\right]$. The imaginary part of $v_{m}(k, v)$ will be nonzero if and only if the term under the inner square root in (14) is negative. Thus, to find $v_{m}^{*}$, we need to solve:

$$
\left[G_{m}\left(k, v=v_{m}^{*}\right)\right]^{2}-64 k^{4}\left(v_{m}^{*}\right)^{4}=0 .
$$



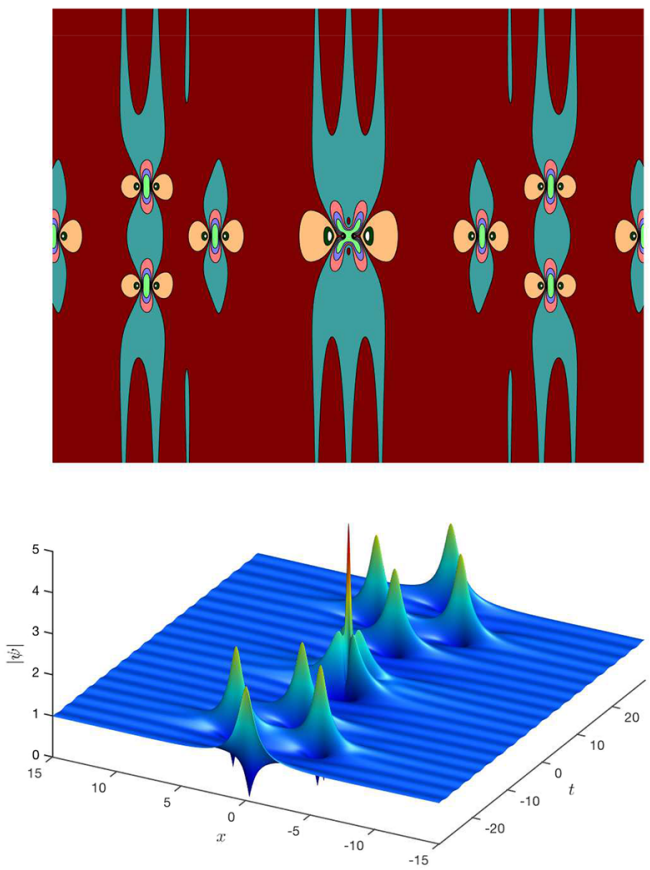

(a)
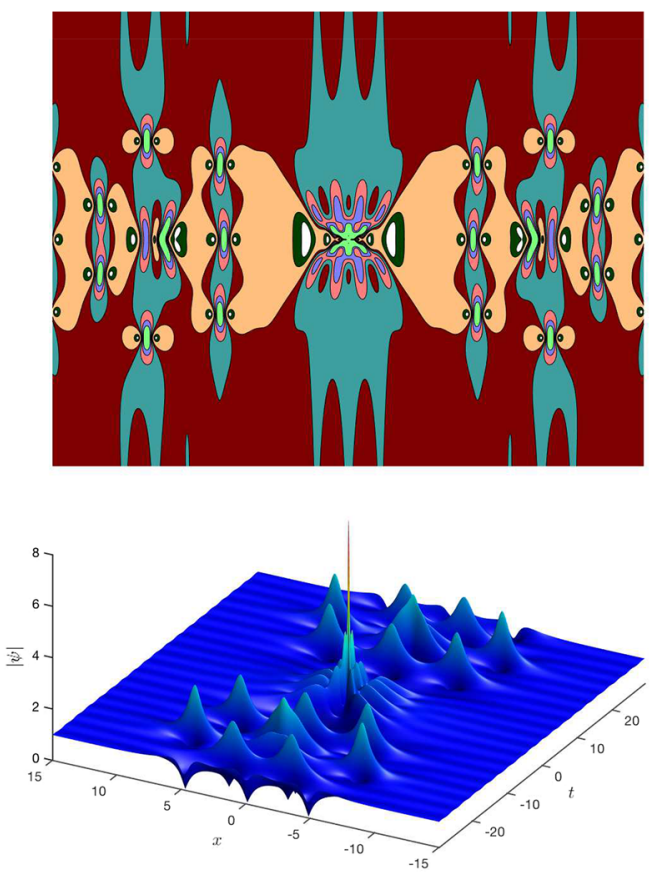

(c)

Fig. 2 Higher-order breathers on a dn background with $k=1 / 4$ and $\kappa_{m} \in \mathbb{R}$. a Second-order, with $\left\{v_{m}\right\}=\{0.98,0.91\}$. b Third-order, with $\left\{v_{m}\right\}=\{0.98,0.91,0.84\} \mathbf{c}$ Fourth-order, with
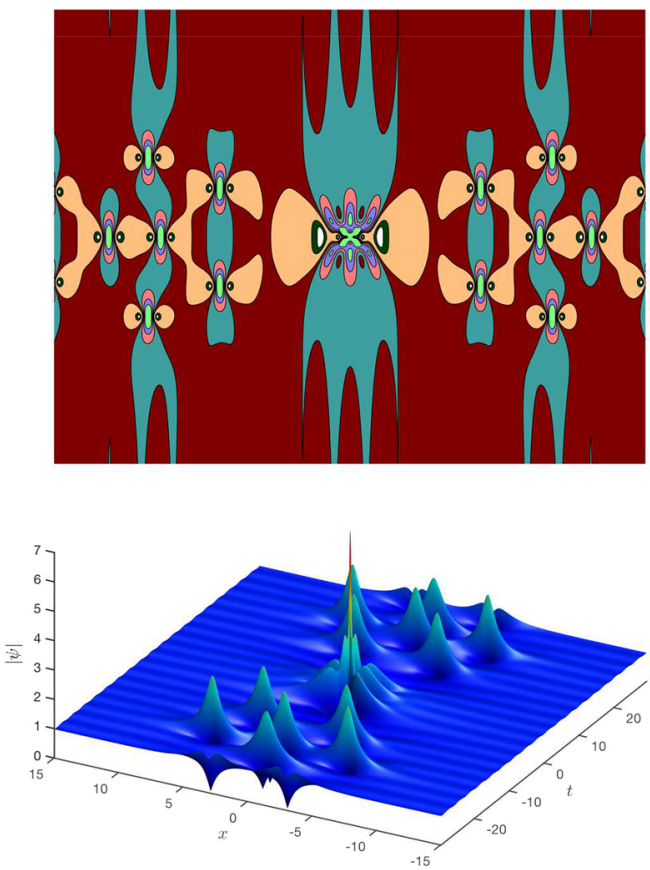

(b)
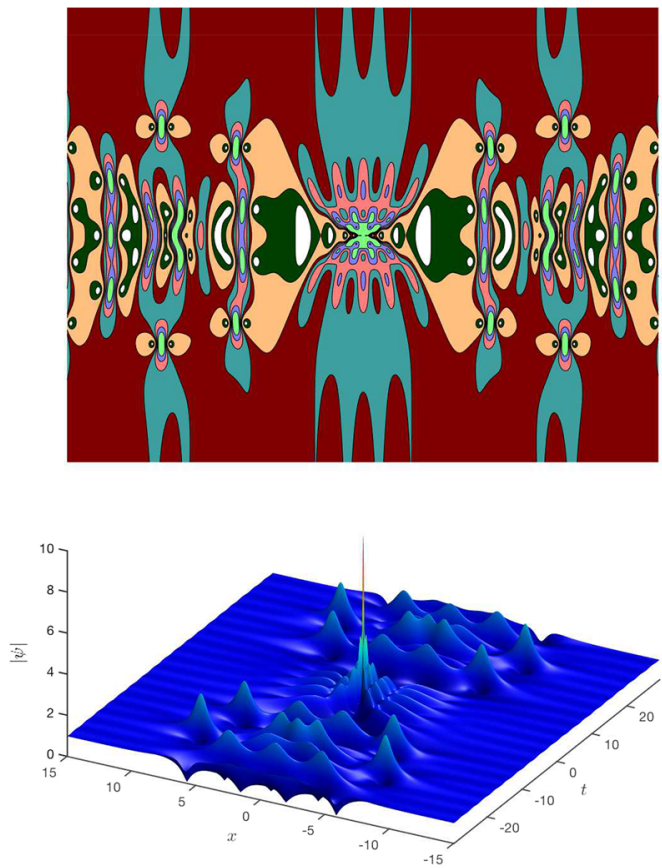

(d)

$\left\{v_{m}\right\}=\{0.98,0.91,0.84,0.72\}$ d Fifth-order, with $\left\{v_{m}\right\}=$ $\{0.98,0.91,0.84,0.72,0.51\}$. Inserts: Contour plots to emphasize the low- $|\psi|$ details 
Fig. 3 Plot of the eigenvalues $v_{m}$ given by (14) as a function of $v$, with $k=2 / 5$. The dashed vertical lines represent the lower limit $v_{m}^{*}$ (Eq. 18), and the horizontal lines give the upper and lower limit on $v_{m}$

Fig. 4 Plot of the peak height formula (5) at $k=1 / 2$ using the eigenvalues given by (14). The insert shows a zoomed-in version of the region between the peak heights of first- and second-order breathers, with a gap of $\Delta \psi(0,0)=1 / 2=k$
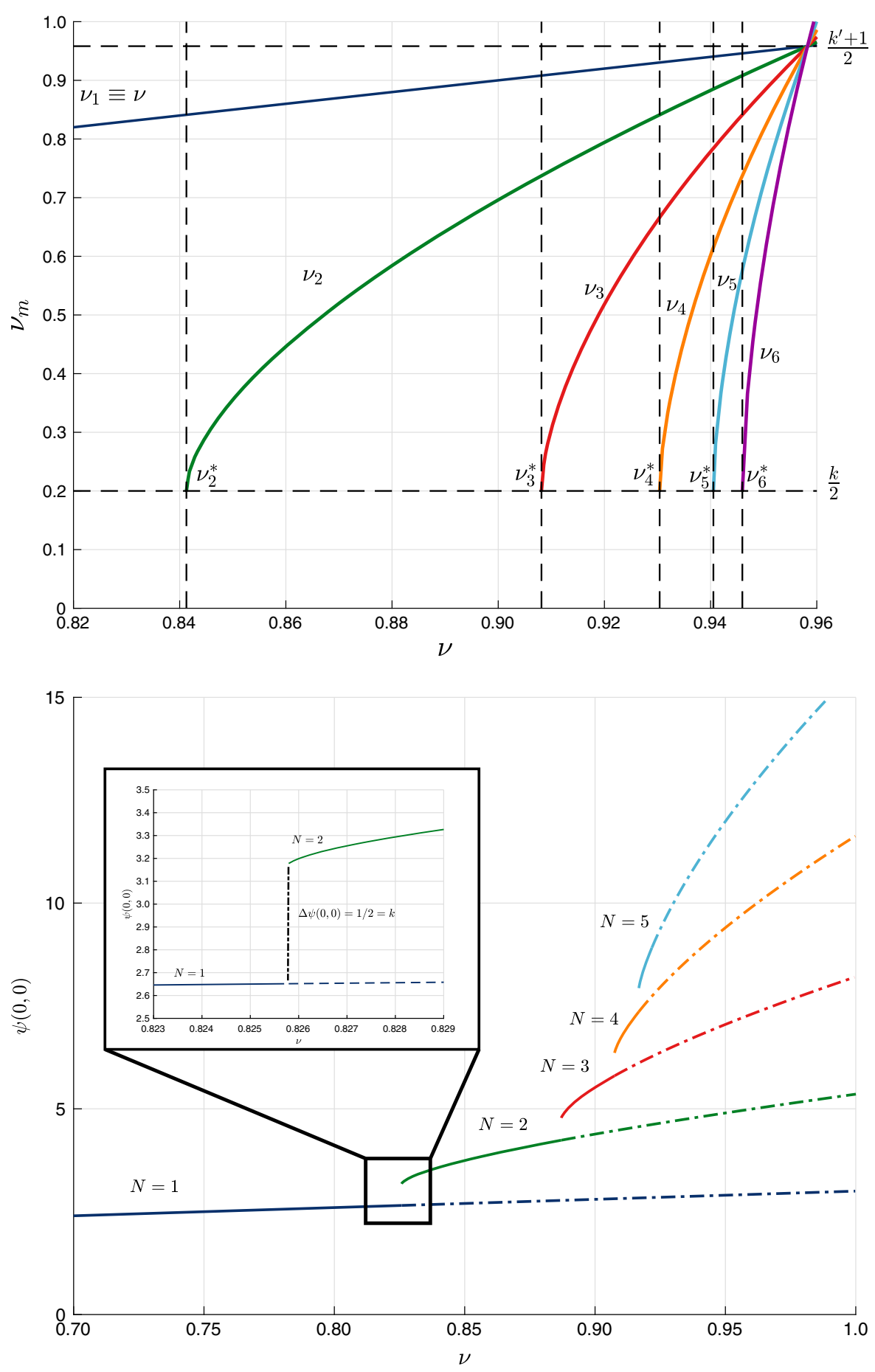

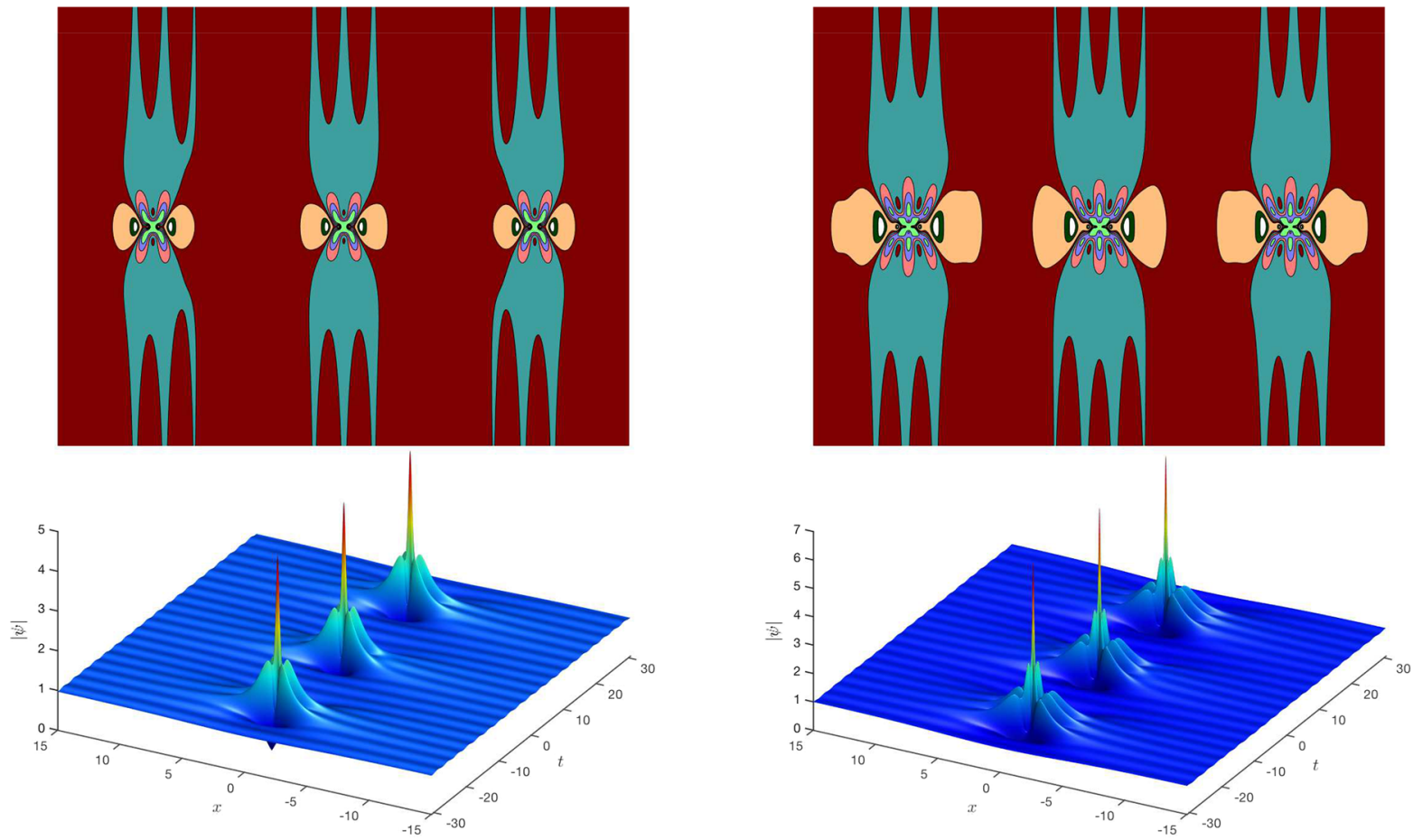

(a)

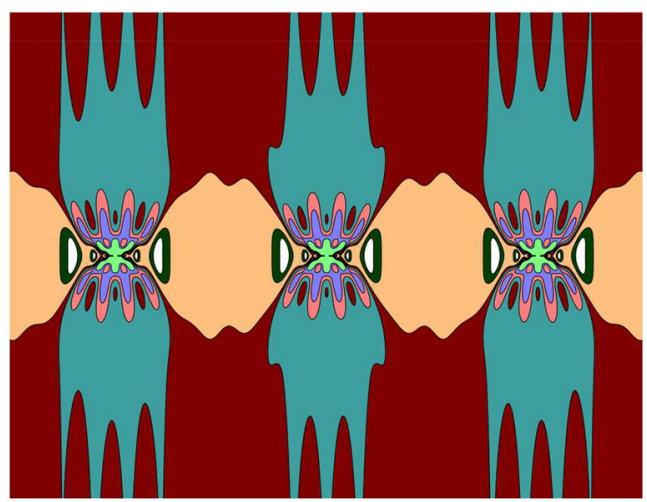

(b)

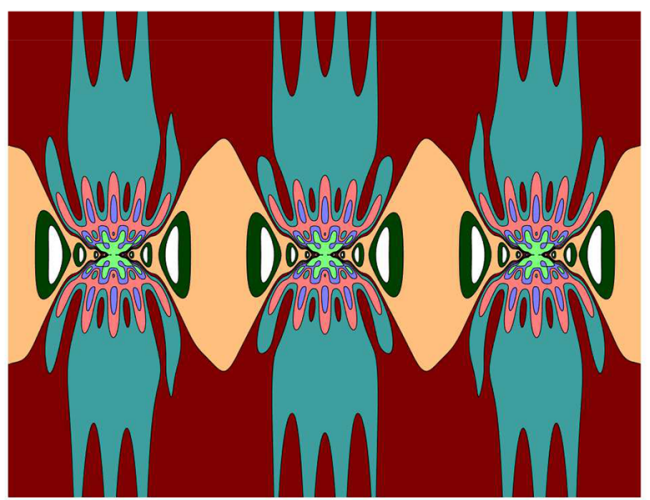

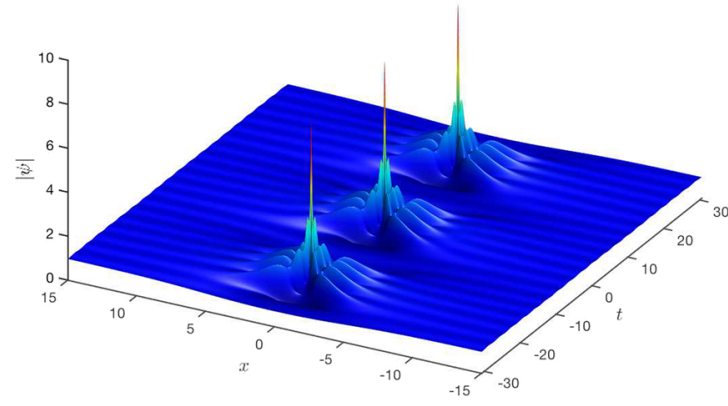

(c)

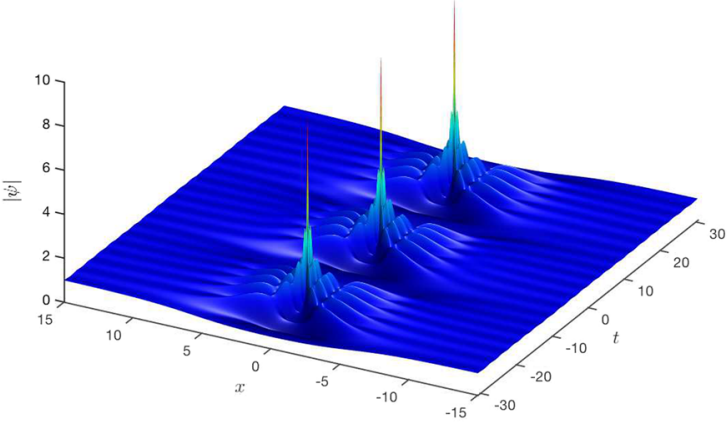

(d)

Fig. 5 Quasiperiodic higher-order breathers with ( $v \approx 0.972, k=1 / 4)$, and higher-order $v_{m}$ computed via Eq. (14) a second order $\mathbf{b}$ third order $\mathbf{c}$ fourth order $\mathbf{d}$ fifth order. Inserts: contour plots to emphasize the low- $|\psi|$ details 
Fig. $6 v k$-plane, showing different regions of periodicity. The light blue shaded region is where $\kappa$ is real, given by (11). The solid curves correspond to different values of $q$ in (23)

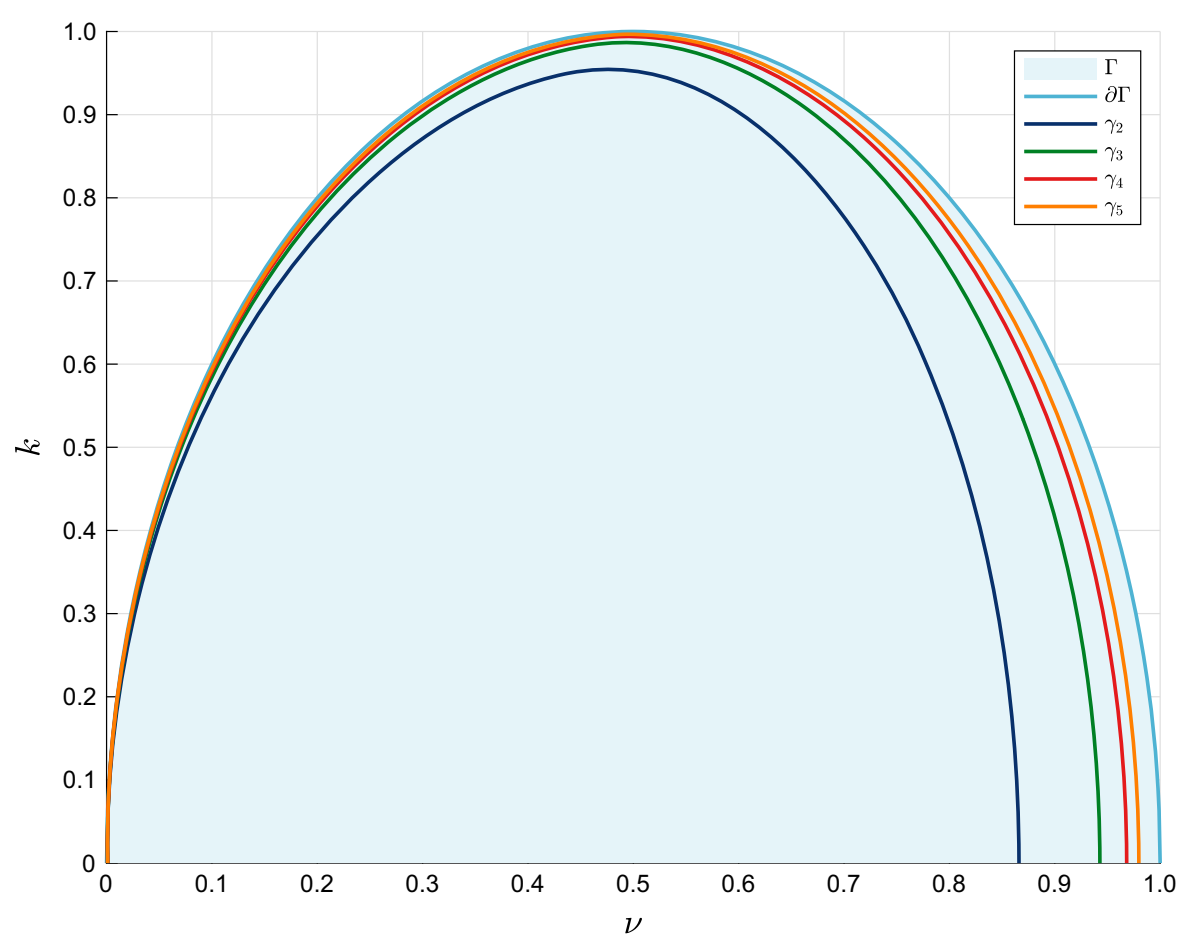

This gives:

$v_{m}^{*}(k)=\frac{\sqrt{2 C_{m}(k)+H_{m}(k)}}{2 m}$,

where

$$
\begin{aligned}
& C_{m}(k)=\sqrt{\left(m^{2}-1\right)\left(k^{\prime}\right)^{2}\left(m^{2}-\left(k^{\prime}\right)^{2}\right)}, \\
& H_{m}(k)=m^{2}\left(\left(k^{\prime}\right)^{2}+1\right)-2\left(k^{\prime}\right)^{2} .
\end{aligned}
$$

Substituting (18) into (14), one obtains $v_{m}(v=$ $\left.v_{m}^{*}, k\right)=k / 2, \forall m \in \mathbb{Z}^{+}$, independent of $m$. The importance of this result will be discussed in Appendix 1.

Consequently, each curve $v_{m}$ in the $v v_{m}$-plane starts from the point:

$L_{m}(k)=\left(v_{m}^{*}(k), \frac{k}{2}\right) \quad, \quad m \geq 2$.

This is a stricter condition on $v$ than the lower limit of Eq. (8), since $k>\left(1-\sqrt{1-k^{2}}\right) \forall k \in(0,1)$. Referring to the PHF (5), the lower limit (20) then implies that when transitioning from an $N$ th order to an $(N+1)$ st order breather, the peak would instantly jump by $2(k / 2)=k$ and would not just increase smoothly as in the simpler constant background case [18]. This can be seen clearly in Fig. 4.

Figure 5 shows the three "quasi-periods" of higherorder breathers with $v_{m}$ obtained from Eq. (14), and $v$ and $k$ selected arbitrarily. The effect of matching the constituent breathers' periods drastically improves the overall periodicity of the high-order breather, with seemingly repeating higher-order side peaks in the 3D plots. However, closer inspection of contour plots near the breather's base reveals remaining distortions in the side peaks. They are still not identical to the central peak. This distortion is reminiscent of the first-order case, as shown in Fig. 1. These higher-order breathers are all quasiperiodic. In the limit of $k \rightarrow 0, v_{m} \rightarrow$ $\sqrt{m^{2}-1} / m,(14)$ reproduces our previous results of $a_{m}=v_{m}^{2} / 2=\left(1-1 / m^{2}\right) / 2$ for breathers on a constant background in Ref. [18].

\section{Fully periodic breathers}

The period of the $\operatorname{dn}(t ; k)$ background is given by

$T_{\mathrm{dn}}=2 K(k)$ 
Fig. 7 Fully periodic first-order breathers with $(v \approx 0.848, k=3 / 5) \in \gamma_{3}$
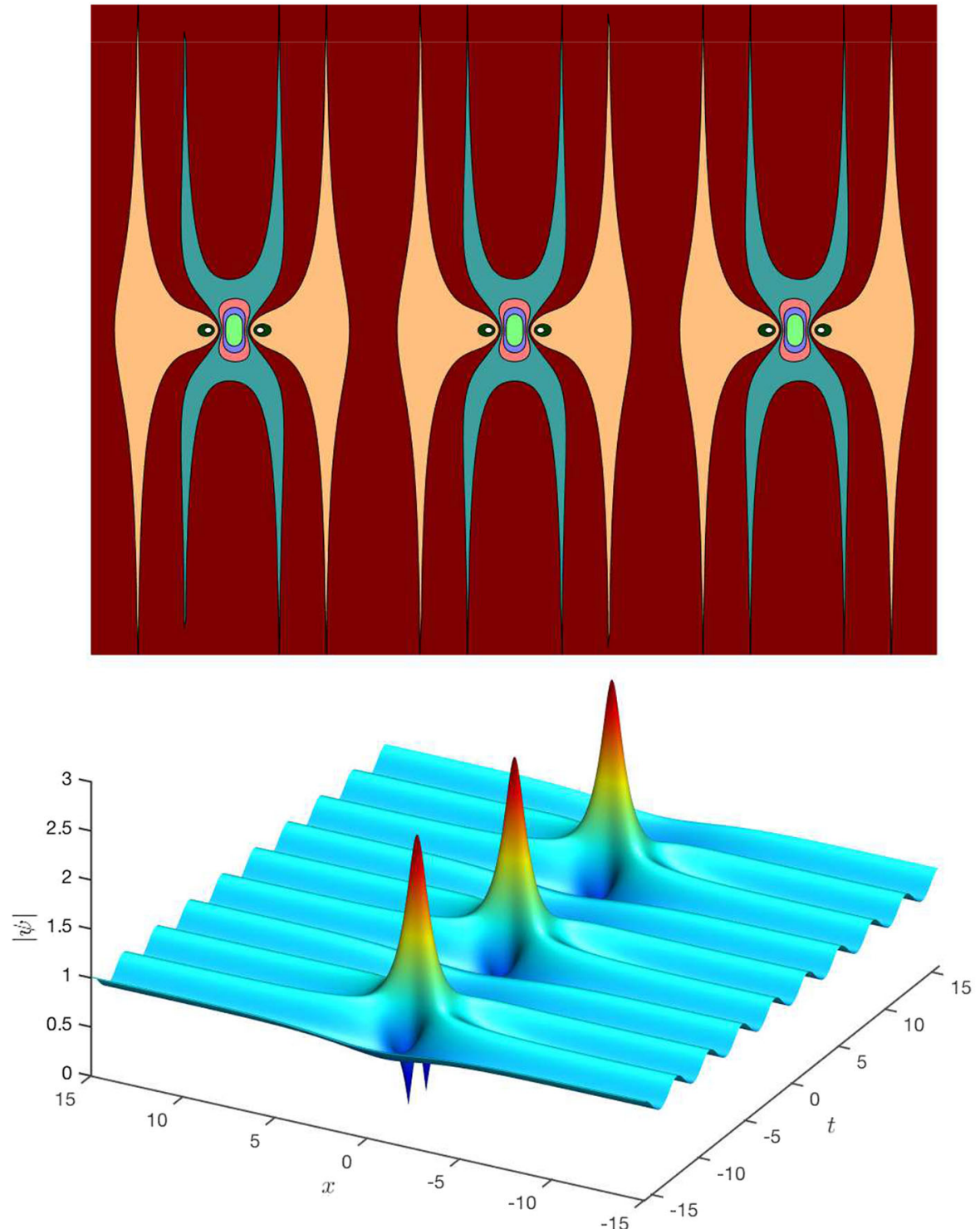

Substituting back into (6), one obtains the condition for matching a breather to the background:

$$
\begin{gathered}
(\nu, k) \in \gamma_{q}:=\{(v, k) \in(0,1) \times[0,1] \\
\left.\left(\frac{\pi}{2 q K(k)}\right)^{2}+\frac{\left(k^{2}+4 v^{2}\right)^{2}}{16 v^{2}}=1\right\} .
\end{gathered}
$$

Note that $\gamma_{q=1}=\varnothing$ (i.e., one cannot match to the breather so that its period is exactly equal to the background, it must be a periodic multiple). As shown in Fig. 6, it is clear that $\gamma_{q} \subset \Gamma \forall q \in \mathbb{Z}^{+}$, and $\gamma_{q \rightarrow \infty}=$

$\kappa=\frac{\pi}{2 q K(k)}$. 

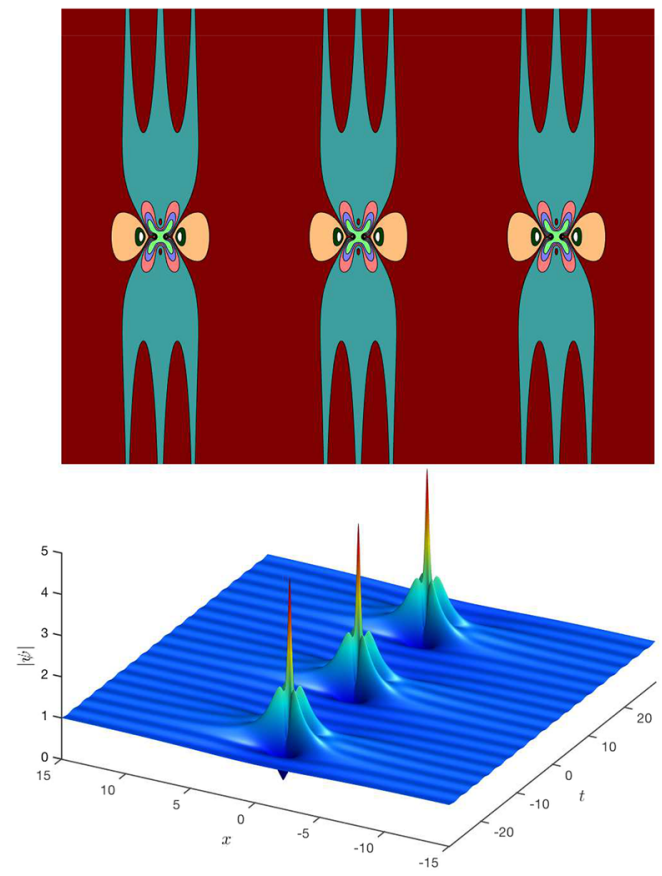

(a)
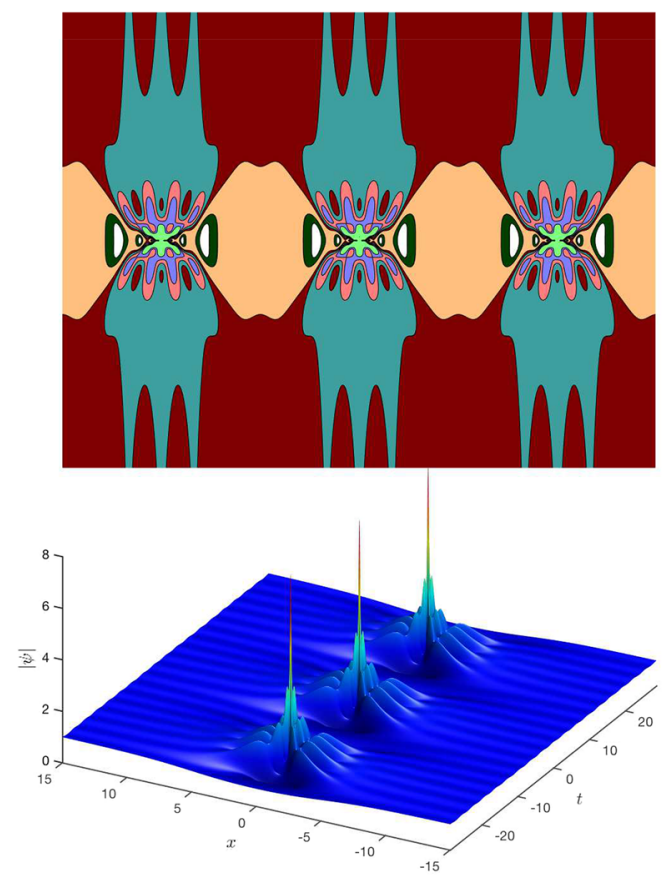

(c)

Fig. 8 Fully periodic higher-order breather with $(v \approx$ $0.9704, k=1 / 4) \in \gamma_{6}$, and the higher $v_{m}$ calculated via Eq. (14). a Second order. b Third order $\mathbf{c}$ Fourth order. d Fifth order.
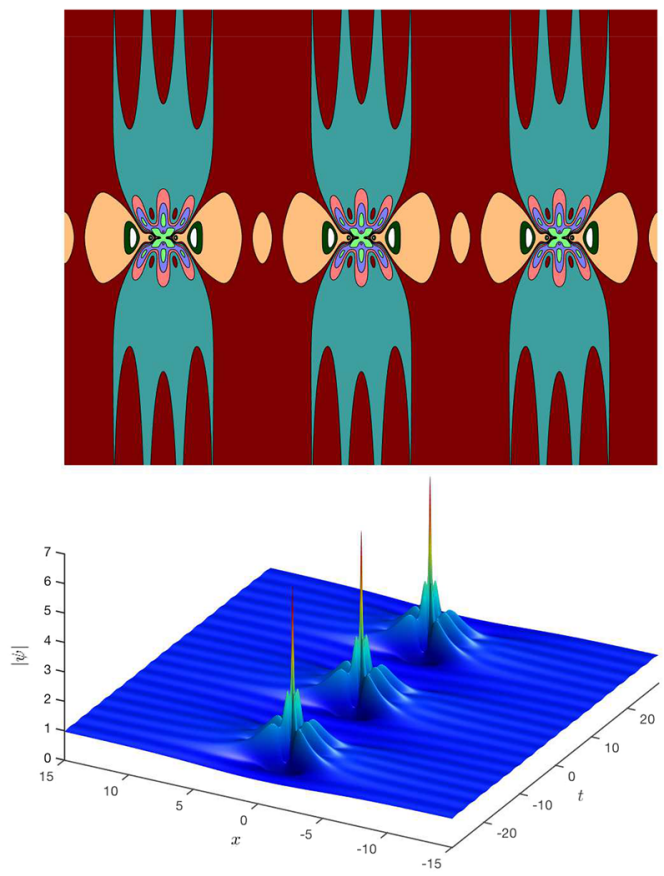

(b)
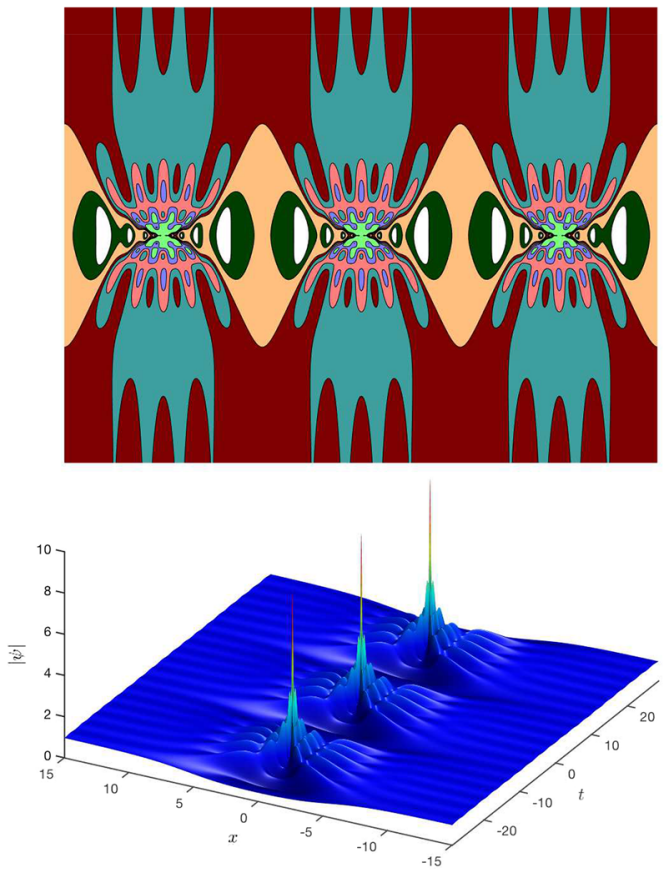

(d)

Insert: Contour plots to emphasize the low- $|\psi|$ detail. The symmetry between the central and side peaks is apparent 
Table 1 Necessary and sufficient conditions on the parameters for a fully periodic breather of order $N$

\begin{tabular}{lll}
\hline Condition & Equation & Explanation \\
\hline$(v, k) \in \gamma_{q}, q=2,3, \ldots$ & Eq. (23) & Matches a breather to the background \\
$v \equiv v_{1}>v_{N}^{*}$ & Eq. (18) & Ensures $v_{m} \in \mathbb{R}, m=1, \ldots, N$ \\
$v_{m}=v_{m}(v, k), m=1, \ldots, N$ & Eq. (14) & Matches the constituent breathers to each other \\
\hline
\end{tabular}

$\partial \Gamma$ (i.e., the boundary of $\Gamma$, Eq. (11)). Thus, any $(v, k) \in \gamma_{q}$ results in $\kappa \in \mathbb{R}$. Additionally, if a higherorder breather has matched constituents via Eq. (14), its period will be $T_{B}$, and thus one only needs $(v, k) \in \gamma_{q}$.

Figure 7 shows a fully periodic first-order breather. It is clear that selecting a set of parameters $(v, k)$ satisfying Eq. (23) leads to a truly periodic structure. We additionally show several fully periodic higher-order breathers in Fig. 8, combining conditions (23) and (14). In general, the effect of matching to the background is subtle in $3 \mathrm{D}$ plots yet abundantly clear in the contour plots. The central breather's peak is centered at the origin, where $\psi_{0}(0,0)=1$. If the parameters $(\nu, k) \notin \gamma_{q}$ (i.e., $\left.q \notin \mathbb{Z}\right)$, the side peaks will then be displaced from the peak of $\psi_{0}$. On the other hand, when $(\nu, k) \in \gamma_{q}, q \in \mathbb{Z}^{+}$, all breather peaks lie precisely on top of the background crests, and we get perfect periodicity. We provide a video in this work's supplemental material, which visually demonstrates this process.

\section{Discussion and conclusion}

In this work, we have numerically solved for the higherorder breathers of the nonlinear Schrödinger equation on an elliptic background by iterating the Darboux transformation. We find that the periodic background exerts a much more profound impact on the higherorder breathers than previously thought. Because of the periodic background, any unmatched higher-order breather has only a single high-intensity peak, resembling a rogue wave. These QRWs have a far richer structure than the standard, monotonic Peregrine-like rational rogue waves.

The chaotic background shown in Fig. 2 is also of interest. It is well known that two-wave mixing can only result in quasiperiodic structures. This is the case of Fig. 1, the two waves being waves having the breather's period and that of the background. However, three and more wave mixing can lead to chaos. This is the resulting chaotic-looking background we see in Fig. 2.
Truly periodic higher-order breathers can only be recovered by matching all the constituent breathers' periods to each other and the background. Matching constituent breathers' periods to each other produces quasiperiodic with distorted side peaks. Further matching the breathers' periods to that of the background yields side peaks identical to the central peak, sitting precisely on top of the background's crests some periodic distance away. While a general breather of order $N$ has an $N+1$-dimensional parameter space, fully periodic breathers are constrained to a countably infinite set of contours in a 2D parameter space, thus being exceedingly rare. We have termed such a periodic higher-order breather the periodic rogue wave in Ref. [29]. Our findings are summarized in Table 1 . This explains why, on a periodic background, the only common high peak structures that can occur are, paradoxically, the quasirogue waves of Fig. 2.

Acknowledgements This research is supported by the Qatar National Research Fund. O.A.A. is supported by the Berkeley Graduate Fellowship and the Anselmo J. Macchi Graduate Fellowship. S.N.N. acknowledges funding provided by the Institute of Physics Belgrade, through the grant by the Ministry of Education, Science, and Technological Development of the Republic of Serbia. M.R.B. acknowledges support by the Al-Sraiya Holding Group.

Data availability The parameters required to generate the datasets used in the current study are included in the article.

Open Access This article is licensed under a Creative Commons Attribution 4.0 International License, which permits use, sharing, adaptation, distribution and reproduction in any medium or format, as long as you give appropriate credit to the original author(s) and the source, provide a link to the Creative Commons licence, and indicate if changes were made. The images or other third party material in this article are included in the article's Creative Commons licence, unless indicated otherwise in a credit line to the material. If material is not included in the article's Creative Commons licence and your intended use is not permitted by statutory regulation or exceeds the permitted use, you will need to obtain permission directly from the copyright holder. To view a copy of this licence, visit http://creativecommons.org/licenses/by/4.0/. 


\section{A The Darboux transformation and the peak height formula}

The cubic NLSE (1) can be written as the compatibility condition of the following two equations [1]:

$$
R_{t}=\boldsymbol{L} R, \quad R_{x}=\boldsymbol{A} R,
$$

where

$$
\begin{aligned}
& R=\left(\begin{array}{l}
r \\
s
\end{array}\right) \equiv\left(\begin{array}{l}
r_{1 m} \\
s_{1 m}
\end{array}\right), \\
& \boldsymbol{L}=\left(\begin{array}{cc}
-i \lambda & \psi \\
-\psi^{*} & i \lambda
\end{array}\right) \text {, } \\
& \boldsymbol{A}=\left(\begin{array}{lc}
-i \lambda^{2}+i \frac{1}{2}|\psi|^{2} & \lambda \psi+i \frac{1}{2} \psi_{t} \\
-\lambda \psi^{*}+i \frac{1}{2} \psi_{t}^{*} & i \lambda^{2}-i \frac{1}{2}|\psi|^{2}
\end{array}\right) .
\end{aligned}
$$

The operators (matrices) $\boldsymbol{L}$ and $\boldsymbol{A}$ are known as the Lax pair of (1), and the functions $r(x, t)$ and $s(x, t)$ are the Lax pair generating functions. $\lambda$ is generally a complex eigenvalue and is independent of the evolution variable $x$ (so that the Lax pair is isospectral). The compatibility condition of (24) is known as the Lax equation or zerocurvature condition and gives rise to the cubic NLSE (1):

$\boldsymbol{L}_{x}-\boldsymbol{A}_{t}-[\boldsymbol{A}, \boldsymbol{L}]=\mathbf{0}$,

where $[\boldsymbol{A}, \boldsymbol{L}] \equiv \boldsymbol{A} \boldsymbol{L}-\boldsymbol{L} \boldsymbol{A}$ is the commutator.

Given an initial solution of (1), one can find a more complicated solution of order $N$ via the Darboux transformation: $[1,8,30,31]$ :

$$
\psi_{N}=\psi_{0}+\sum_{m=1}^{N} \frac{2 i r_{m 1} s_{m 1}^{*}\left(\lambda_{m}-\lambda_{m}^{*}\right)}{\left|r_{m 1}\right|^{2}+\left|s_{m 1}\right|^{2}},
$$

where the higher-order Lax-pair generating functions are computed recursively via $[1,31]$ :

$$
\begin{aligned}
r_{m j} & =\left[\left(l_{m-1}^{*}-l_{m-1}\right) s_{m-1,1}^{*} r_{m-1,1} s_{m-1, j+1}\right. \\
& +\left(l_{j+m-1}-l_{m-1}\right)\left|r_{m-1,1}\right|^{2} r_{m-1, j+1} \\
& \left.+\left(l_{j+m-1}-l_{m-1}^{*}\right)\left|s_{m-1,1}\right|^{2} r_{m-1, j+1}\right] \\
& /\left(\left|r_{m-1,1}\right|^{2}+\left|s_{m-1,1}\right|^{2}\right), \\
s_{m j} & =\left[\left(l_{m-1}^{*}-l_{m-1}\right) s_{m-1,1} r_{m-1,1}^{*} r_{m-1, j+1}\right. \\
& +\left(l_{j+m-1}-l_{m-1}\right)\left|s_{m-1,1}\right|^{2} s_{m-1, j+1} \\
& \left.+\left(l_{j+m-1}-l_{m-1}^{*}\right)\left|r_{m-1,1}\right|^{2} s_{m-1, j+1}\right]
\end{aligned}
$$

$$
/\left(\left|r_{m-1,1}\right|^{2}+\left|s_{m-1,1}\right|^{2}\right) .
$$

To find the starting functions of the recursion, i.e., $r_{1 m}$ and $s_{1 m}$, we take the Ansätze:

$$
\begin{gathered}
r_{1 m}(x, t)=a_{1 m}(x, t) e^{\frac{i x}{4}\left(k^{2}-2\right)}, \\
s_{1 m}(x, t)=a_{1 m}(x, t) e^{-\frac{i x}{4}\left(k^{2}-2\right)},
\end{gathered}
$$

and $\psi$ as given in Eq. (2). Substituting in the Lax pair Eq. (24) and suppressing subscripts, one gets [9]

$$
\begin{aligned}
a_{t} & =i \lambda a(x, t)+i b(x, t) \operatorname{dn}(t ; k), \\
b_{t} & =-i \lambda b(x, t)+i a(x, t) \operatorname{dn}(t ; k), \\
a_{x} & =\frac{1}{2} i a(x, t)\left(2 \lambda^{2}+k^{2}\left(\operatorname{sn}^{2}(t ; k)-\frac{1}{2}\right)\right) \\
& +b(x, t)\left(i \lambda \operatorname{dn}(t ; k)-\frac{k^{2}}{2} \operatorname{sn}(t ; k) \operatorname{cn}(t ; k)\right), \\
b_{x} & =-\frac{1}{2} i b(x, t)\left(2 \lambda^{2}+k^{2}\left(\operatorname{sn}^{2}(t ; k)-\frac{1}{2}\right)\right) \\
& +a(x, t)\left(i \lambda \operatorname{dn}(t ; k)+\frac{k^{2}}{2} \operatorname{sn}(t ; k) \operatorname{cn}(t ; k)\right) .
\end{aligned}
$$

However, as noted in [9], the four coupled first-order differential Eq. (32) cannot be solved analytically. Nonetheless, one can solve for the profiles and derivatives at $t=0$, given by [9]

$$
\begin{aligned}
\left.a_{1 m}\right|_{t=0}= & A e^{i\left(\chi_{m}+\kappa_{m} \lambda_{m}\left(x-x_{m}\right)\right)} \\
& -B e^{-i\left(\chi_{m}+\kappa_{m} \lambda_{m}\left(x-x_{m}\right)\right)}, \\
\left.b_{1 m}\right|_{t=0}= & A e^{i\left(-\chi_{m}+\kappa_{m} \lambda_{m}\left(x-x_{m}\right)\right)} \\
& +B e^{-i\left(-\chi_{m}+\kappa_{m} \lambda_{m}\left(x-x_{m}\right)\right)}, \\
\left.a_{1 m, t}\right|_{t=0}= & i\left(\left.\lambda_{m} a_{1 m}\right|_{t=0}+\left.b_{1 m}\right|_{t=0}\right), \\
\left.b_{1 m, t}\right|_{t=0}= & -i\left(\left.\lambda_{m} b_{1 m}\right|_{t=0}-\left.a_{1 m}\right|_{t=0}\right),
\end{aligned}
$$

where

$\kappa_{m}=\sqrt{\left(\lambda_{m}-\frac{k^{2}}{4 \lambda_{m}}\right)^{2}+1}$

is half the wavenumber of the mth constituent breather, $\chi_{m}=\arccos \left(\kappa_{m}\right) / 2$ is the background-dependent phase, and $A$ and $B$ are two phase constants. One can then evolve Eq. (33) along the $t$-axis numerically. All 
the results in this work employ a fourth-order RungeKutta method and sufficiently small grid spacing.

In previous works $[18,23,24]$, using the DT, we derived the so-called peak height formula (PHF) for $N^{\text {th }}$-order solutions of nonlinear Schrödinger-type equations:

$\psi_{N}(0,0)=\psi_{0}(0,0)+2 \sum_{m=1}^{N} v_{m}$

This requires the Lax pair generating functions to only differ by a phase at the origin [23]:

$s_{1 m}(0,0)=e^{i \phi} r_{1 m}(0,0)$,

One can easily verify that equations (33), with proper choice of $A$ and $B$, lead to $r(x, t)$ and $s(x, t)$ that obey the PHF condition (36) with $\phi=\pi / 2$, leading to the PHF (5).

\section{References}

1. Akhmediev, N.N., Ankiewicz, A.: Solitons Nonlinear Pulses and Beams. Chapman \& Hall, London (1997)

2. Solli, D.R., Ropers, C., Koonath, P., Jalali, B.: Optical rogue waves. Nature 450(13), 1054-1057 (2007)

3. Frisquet, B., Kibler, B., Millot, G.: Collision of Akhmediev breathers in nonlinear fiber optics. Phys. Rev. X 3(4), 041032 (2014)

4. Armaroli, A., Conti, C., Biancalana, F.: Rogue solitons in optical fibers: a dynamical process in a complex energy landscape? Optica 2(5), 497 (2015)

5. Onorato, M., Residori, S., Bortolozzo, U., Montina, A., Arecchi, F.T.: Rogue waves and their generating mechanisms in different physical contexts. Phys. Rep. 528(2), 47-89 (2013)

6. Akhmediev, N., Soto-Crespo, J.M., Ankiewicz, a: How to excite a rogue wave. Phys. Rev. A 80(4), 043818 (2009)

7. Kedziora, D.J., Ankiewicz, A., Akhmediev, N.: Triangular rogue wave cascades. Phys. Rev. E 86(5), 056602 (2012)

8. Kedziora, D.J., Ankiewicz, A., Akhmediev, N.: Circular rogue wave clusters. Phys. Rev. E 84(056611), 056611 (2011)

9. Kedziora, D.J., Ankiewicz, A., Akhmediev, N.: Rogue waves and solitons on a cnoidal background. Eur. Phys. J. Spec. Top. 223(1), 43-62 (2014)

10. Kedziora, D.J., Ankiewicz, A., Akhmediev, N.: Secondorder nonlinear Schrödinger equation breather solutions in the degenerate and rogue wave limits. Phys. Rev. E 85(6), 066601 (2012)

11. Akhmediev, N., Ankiewicz, A., Soto-Crespo, J.M.: Rogue waves and rational solutions of the nonlinear Schrödinger equation. Phys. Rev. E 80(2), 026601 (2009)
12. Dudley, J.M., Dias, F., Erkintalo, M., Genty, G.: Instabilities, breathers and rogue waves in optics. Nat. Photonics 8(September), 755-764 (2014)

13. Fleischhauer, M., Imamoglu, A., Marangos, J.P.: Electromagnetically induced transparency: optics in coherent media. Rev. Mod. Phys. 77, 633-673 (2005)

14. Nikolić, S.N., Radonjić, M., Krmpot, A.J., Lučić, N.M., Zlatković, B.V., Jelenković, B.M.: Effects of a laser beam profile on Zeeman electromagnetically induced transparency in the Rb buffer gas cell. J. Phys. B At. Mol. Opt. Phys. 46, 075501 (2013)

15. Krmpot, A.J., Ćuk, S.M., Nikolić, S.N., Radonjić, M., Slavov, D.G., Jelenković, B.M.: Dark Hanle resonances from selected segments of the Gaussian laser beam cross-section. Opt. Express 17, 22491-22498 (2009)

16. Li, Z.-Y., Li, F.-F., Li, H.-J.: Exciting rogue waves, breathers, and solitons in coherent atomic media. Commun. Theor. Phys. 72, 075003 (2020)

17. Chin, S.A., Ashour, O.A., Belić, M.R.: Anatomy of the Akhmediev breather: cascading instability, first formation time, and Fermi-Pasta-Ulam recurrence. Phys. Rev. E 92(6), 063202 (2015)

18. Chin, S.A., Ashour, O.A., Nikolić, S.N., Belić, M.R.: Maximal intensity higher-order Akhmediev breathers of the nonlinear Schrödinger equation and their systematic generation. Phys. Lett. A 380(43), 3625-3629 (2016)

19. Ankiewicz, A., Kedziora, D.J., Akhmediev, N.: Rogue wave triplets. Phys. Lett. A 375(28-29), 2782-2785 (2011)

20. Chowdury, A., Kedziora, D.J., Ankiewicz, A., Akhmediev, N.: Breather-to-soliton conversions described by the quintic equation of the nonlinear Schrödinger hierarchy. Phys. Rev. E 91(3), 032928 (2015)

21. Ankiewicz, A., Kedziora, D.J., Chowdury, A., Bandelow, U., Akhmediev, N.: Infinite hierarchy of nonlinear Schrödinger equations and their solutions. Phys. Rev. E 93(1), 012206 (2016)

22. Kedziora, D.J., Ankiewicz, A., Chowdury, A., Akhmediev, N.: Integrable equations of the infinite nonlinear Schrödinger equation hierarchy with time variable coefficients. Chaos 25(10), 17105 (2015)

23. Chin, S.A., Ashour, O.A., Nikolić, S.N., Belić, M.R.: Peakheight formula for higher-order breathers of the nonlinear Schrödinger equation on non-uniform backgrounds. Phys. Rev. E 95, 012211 (2017)

24. Nikolić, S.N., Aleksić, N.B., Ashour, O.A., Belić, M.R., Chin, S.A.: Systematic generation of higher-order solitons and breathers of the Hirota equation on different backgrounds. Nonlinear Dyn. 89(3), 1637-1649 (2017)

25. Ashour, O.A.: Maximal Intensity Higher-Order Breathers of the Nonlinear Schrödinger Equation on Different Backgrounds. Undergraduate Research Scholars Thesis, Texas A\&M University (2017)

26. Akhmediev, N.N., Korneev, V.I.: Modulation instability and periodic solutions of the nonlinear Schrödinger equation. Teor. i. Mat. Fiz. 69(2), 189-194 (1987)

27. Kuznetsov, E.: Solitons in a parametrically unstable plasma. Akad. Nauk SSSR Dokl. 236, 575-577 (1977)

28. Ma, Y.C.: The perturbed plane-wave solutions of the Cubic Schrödinger equation. Stud. Appl. Math. 60(1), 43-58 (1979) 
29. Nikolić, S.N., Ashour, O.A., Aleksić, N.B., Belić, M.R., Chin, S.A.: Breathers, solitons and rogue waves of the quintic nonlinear Schrödinger equation on various backgrounds. Nonlinear Dyn. 95, 2855-2865 (2019)

30. Akhmediev, N., Soto-crespo, J.M., Ankiewicz, A.: Extreme waves that appear from nowhere: on the nature of rogue waves. Phys. Lett. A 373(25), 2137-2145 (2009)
31. Matveev, V.B., Salle, M.A.: Darboux Transformations and Solitons. Springer-Verlag, Heidelberg (1991)

Publisher's Note Springer Nature remains neutral with regard to jurisdictional claims in published maps and institutional affiliations. 